\title{
Philosophiques
}

\section{Émergence et incorporation selon Margolis}

\section{Dale Jacquette}

Volume 13, numéro 1, printemps 1986

URI : https://id.erudit.org/iderudit/203303ar

DOI : https://doi.org/10.7202/203303ar

Aller au sommaire du numéro

Éditeur(s)

Société de philosophie du Québec

ISSN

0316-2923 (imprimé)

1492-1391 (numérique)

Découvrir la revue

Citer cet article

Jacquette, D. (1986). Émergence et incorporation selon Margolis. Philosophiques, 13(1), 53-63. https://doi.org/10.7202/203303ar

\section{Résumé de l'article}

L'analyse de la personne ou de l'oeuvre d'art comme entité matériellement incorporée et culturellement émergente est centrale dans l'oeuvre de Joseph Margolis : il tente de développer un dualisme des propriétés, comme alternative au réalisme platonicien et au dualisme ontique cartésien, en esthétique et en " philosophy of mind ». La définition de l'incorporation proposée par Margolis est ici critiquée par le moyen d'un contre-exemple, et plusieurs révisions possibles de cette définition, en vue d'éviter l'objection, sont aussi rejetées. La théorie de Margolis nous délivre en principe des inconvénients métaphysiques des ontologies traditionnelles ; mais elle ne peut être acceptée tant et aussi longtemps que le concept d'incorporation n'aura pas été défini d'une façon satisfaisante. 


\title{
ÉMERGENCE ET INCORPORATION SELON MARGOLIS
}

\author{
par Dale Jacquette *
}

\begin{abstract}
RESUMÉ. L'analyse de la personne ou de l'œuvre d'art comme entité matériellement incorporée et culturellement émergente est centrale dans l'œuvre de Joseph Margolis: il tente de développer un dualisme des propriétés, comme alternative au réalisme platonicien et au dualisme ontique cartésien, en esthétique et en " philosophy of mind». La définition de l'incorporation proposée par Margolis est ici critiquée par le moyen d'un contre-exemple, et plusieurs révisions possibles de cette définition, en vue d'éviter l'objection, sont aussi rejetées. La théorie de Margolis nous délivre en principe des inconvénients métaphysiques des ontologies traditionnelles; mais elle ne peut être acceptée tant et aussi longtemps que le concept d'incorporation n'aura pas été défini d'une façon satisfaisante.
\end{abstract}

ABSTRACT. The analysis of a person or artwork as a materially embodied culturally emergent entity is central to Joseph Margolis' attempt to develop property dualism as an alternative to Platonic realism or ontic Cartesian dualism in aesthetics and the philosophy of mind. Margolis' definition of material embodiment is criticized by counter-example, and several methods of revising the definition to avoid the objection are discredited. Margolis' theory in principle offers relief from the metaphysical disadvantages of traditional ontologies. But the proposal cannot be accepted unless or until the concept of material embodiment is correctly defined.

L'analyse des personnes ou des œuvres d'art en tant qu'entités matériellement incorporées et culturellement émergentes constitue le noud central de certaines contributions récentes de Joseph Margolis à l'esthétique et à la «philosophy of mind».

* Ce texte a été présenté lors d'un congrès de l'American Society for Aesthetics, Eastern Division, State University of New York, Buffalo, le 24 mars 1984. L'auteur remercie Mme Juliette Veilleux pour son excellente traduction française. 
Dans Persons and Minds: The Prospects of Nonreductive Materialism, Art and Philosophy, Culture and Cultural Entities: Toward a New Unity of Science, Philosophy of Psychology et certains essais connexes, Margolis tente de se frayer une troisième voie entre, d'une part, les ontologies traditionnelles ontiquement dualistes (cartésiennes) ou réalistes (platoniciennes), et d'autre part, les ontologies matérialistes réductrices. Sa théorie met en avant un dualisme d'attributs, de propriétés matérielles et intentionnelles distinctes, au lieu d'un dualisme ontique de substances matérielles et immatérielles distinctes.

Les œuvres d'art et les personnes sont matériellement incorporées mais non réductibles, et donc pas identiques, à leurs incorporations matérielles ${ }^{1}$. Elles sont distinctes du matériau physique dans lequel elles sont incorporées parce qu'elles possèdent des propriétés intentionnelles qui ne peuvent être expliquées en termes physiques, matériels ou mécaniques, dans un langage purement extensionnel ${ }^{2}$. Pour les entités culturellement émergentes, Margolis propose des critères fonctionnels d'identité qui ne sont pas abstraits, mais physiquement réalisés et incarnés, et il définit les fonctions incarnées et individuantes comme des intentionnalités linguistiques, psychologiques et esthétiques irréductibles ${ }^{3}$.

L'alternative que propose Margolis pour l'interprétation des personnes et des cuvres d'art prétend éviter certaines difficultés classiques des métaphysiques exclusivement matérialistes et non matérialistes. Mais en raison de leur importance pour la solution de ces problèmes, ses concepts d'incorporation matérielle et d'émergence culturelle doivent être soumis à un examen critique sérieux.

1. Joseph Margol.1s, Persons and Minds: The Prospects of Nonreductive Materialism, Boston Studies in the Philosophy of Science, Vol. LVII (Dordrecht-Boston : D. Reidel Publishing Company 1978), pp. 16-19, 234-236. Margolis, "Works of Art as Physically Embodied and Culturally Emergent Entities», The British Journal of Aesthetics, Vol. XIV, 1974, p. 187-189.

2. Persons and Minds, p. 18-19.

3. Margolis, Art and Philosophy (Atlantic Highlands: Humanities Press, 1980), p. 2324; Philosophy of Psychology (Englewood Cliffs: Prentice-Hall, Inc. 1984), p. 24-33, 52-55; Culture and Cultural Entities: Toward a New Unity of Science (DordrechtBoston: D. Reidel Publishing Company, 1984), p. 6-7, 124-125; voir aussi Persons and Minds, p. 24-25. 
Dans Persons and Minds, Margolis propose une définition en sept points de l'incorporation:

On a déjà indiqué le sens du terme «incorporation». Cependant, les conditions nécessaires et suffisantes pour l'incorporation d'une entité particulière dans une autre peuvent être formulées de façon plus explicite qu'elles ne l'ont été jusqu'ici: (i) deux entités particulières ainsi reliées ne sont pas identiques; (ii) l'existence de l'entité particulière incorporée présuppose l'existence d'une entité particulière qui l'incorpore (ou incorporante); (iii) l'entité particulière incorporée possède (au moins) quelques-unes des propriétés de l'entité particulière incorporante; (iv) l'entité particulière incorporée possède des propriétés que l'entité particulière incorporante ne possède pas; (v) l'entité particulière incorporée possède une certaine sorte de propriétés que l'entité particulière incorporante ne peut posséder; (vi) l'individuation de l'entité particulière incorporée présuppose l'existence d'une certaine entité particulière incorporante; (vii) l'entité particulière incorporante n'est pas une partie propre de l'entité particulière incorporée 4 .

En regard de la condition (v), il semble que la condition (iv) de Margolis ne soit pas nécessaire. $\mathrm{Si}$, comme le requiert la condition (v), le particulier incorporé possède des propriétés que le particulier incorporant ne peut avoir, alors a fortiori le particulier incorporé doit posséder des propriétés que le particulier incorporant ne possède pas. De la même manière, les principes leibnitziens d'identité pourraient cautionner l'élimination de la condition (i) en faveur de la condition (v).

La condition (vi) exige que l'individuation de l'incorporé présuppose l'existence d'un certain incorporant. Mais dans Art and Philosophy et dans "The Ontological Peculiarity of Works of Art », Margolis stipule, dans une version quelque peu différente de la condition (vi), «... que l'individuation de l'incorporé présuppose l'individuation de l'incorporant "s.

Peut-être y aurait-il lieu d'envisager une révision disjonctive de cette condition (vi), qui comporte à la fois une clause d'existence et une clause d'individuation:

4. Ibid., p. 234. La parenthèse contenue dans le libellé de la condition (ii) a été ajoutée par la traductrice.

5. Margolis, «The Ontological Peculiarity of Works of Art », The Journal of Aesthetics and Art Criticism, Vol. XXXVI, 1977, p. 48 (c'est nous qui soulignons) ; Art and Pbilosophy, p. 21. 
(vi') L'individuation du particulier incorporé présuppose soit l'existence d'un certain particulier incorporant, soit l'individuation du particulier incorporant.

L'expression «l'existence d'un certain», dans la condition révisée, est plus faible que l'expression «l'individuation du», car la seconde inclut la première mais l'inverse n'est pas vrai.

\section{III}

La définition que donne Margolis de l'incorporation est inadéquate. Il existe des contre-exemples où toutes les conditions de cette définition sont satisfaites par deux entités qui ne sont pas incorporées l'une dans l'autre.

Soit une vallée $v$ située entre les montagnes adjacentes $m$ et $n$. Admettons que $p$ soit une pente commune à $v$ et à $m$ sous deux descriptions différentes, soit comme pente ascendante de $v$ et pente descendante de $m$. On peut alors émettre les propositions suivantes:

1) La vallée $v$ n'est pas identique à la montagne $m$.

2) L'existence de la vallée $v$ présuppose l'existence de la montagne $m$. (Si les montagnes $m$ et $n$ n'existaient pas, la vallée $v$ n'existerait pas non plus).

3) Il y a au moins quelques propriétés qui sont communes à $v$ et à $m$. (Les deux possèdent la propriété d'avoir la pente $p$ ).

4) Il y a au moins quelques propriétés que $v$ possède mais que $m$ ne possède pas (voir (5) ci-dessous).

5) Il y a au moins quelques propriétés que $v$ possède mais que $m$ ne peut posséder. (La vallée $v$ a, dans son ensemble, la propriété d'être concave plutôt que convexe. Mais $m$ ne peut avoir cette propriété car, alors par définition, elle ne serait pas une montagne).

6) L'individuation de la vallée $v$ présuppose l'existence de la montagne $m$ ou l'individuation de la montagne $m$. (La vallée ne peut être individuée si les montagnes adjacentes ne le sont pas. Ceci exige que les montagnes adjacentes existent).

7) En dernier ressort, la montagne $m$ n'est évidemment pas une partie propre de la vallée $v$. 
La définition de Margolis implique que la vallée $v$ est matériellement incorporée à la montagne $m$. Mais les vallées ne sont pas incorporées à une seule montagne. La vallée n'est pas une entité émergente comportant des propriétés intentionnelles irréductibles. Elle est une entité matérielle tout autant que la montagne et ne peut, selon aucune acception courante de ces termes, être considérée comme émergeant de la montagne, ou incorporée à elle. La vallée $v$ est géologiquement, mais non ontologiquement, différente de la montagne $m$.

Margolis ne prétend pas que toutes les entités matériellement incorporées soient culturellement émergentes; mais tout en admettant quelques recoupements entre elles, il en fait deux catégories distinctes. Pour être culturellement émergente, une entité particulière matériellement incorporée doit aussi comporter des propriétés intentionnelles. Ceci semblerait neutraliser notre contre-exemple, car il est possible que la vallée $v$ soit matériellement incorporée à la montagne $m$ sans en être culturellement émergente. Mais même ceci est inexact. On peut soutenir que la vallée $v$ est matériellement incorporée dans toutes les montagnes environnantes et adjacentes. Mais dans le contre-exemple, la satisfaction des conditions de Margolis implique, sans autre spécification, que la vallée $v$ est matériellement incorporée à la montagne $m$. Pourtant il n'y a tout au plus qu'une partie propre à la vallée $v$ qui est matériellement incorporée à une partie propre de la montagne $m$.

On pourrait objecter que la proposition (6) ne tient pas parce que la montagne $m$ n'a pas besoin d'être pleinement individuée pour pouvoir individuer la vallée $v$. Il pourrait être impossible d'individuer la vallée si la pente $p$ ne l'était pas. Mais $p$ est une partie propre de $v$, de sorte que toute tentative d'avancer un contre-exemple impliquant la vallée $v$ en tant que matériellement incorporée à la pente $p$, serait effectivement bloquée par la condition (vii) de Margolis. Les pentes opposées à $p$ de l'autre côté de $m$ pourraient être reliées à une chaîne de montagnes de façon si complexe que les géographes seraient incapables de s'entendre sur la manière d'individuer $m$ par rapport aux sommets avoisinants de la chaîne, même s'ils n'éprouvaient aucune difficulté à définir, identifier ou individuer la vallée $v$. Dans ce cas, le contre-exemple proposé n'infirmerait 
en rien la définition de Margolis, parce qu'il ne présenterait pas une situation dans laquelle la condition alternative (vi), ou la condition révisée (vi'), serait effectivement satisfaite.

Pour contrer cette objection, on peut reformuler le contreexemple de façon à ce que toutes les conditions de Margolis soient remplies sans équivoque; et pourtant, les mêmes conclusions contraires à l'intuition s'ensuivent, tout comme dans la version originale. Supposons, en effet, que la vallée $v$ ne soit pas simplement flanquée d'un côté par la montagne $m$, mais qu'elle s'enroule complètement autour d'elle, un peu comme la lettre «q» vue d'en haut. Si la vallée était ainsi constituée, il serait impossible d'individuer $v$ sans individuer pleinement $m$. Ceci nous fournit un ensemble de circonstances pour lesquelles $v$ et $m$ satisfont à la fois aux deux formulations de la condition (vi) de Margolis, et à la condition révisée (vi'). Les autres conditions demeurent inchangées et la conclusion inacceptable selon laquelle la vallée est incorporée à la montagne prévaut une fois de plus ${ }^{6}$.

\section{IV}

Comment faire pour amender la définition que donne Margolis de l'incorporation, de façon à éviter ce genre de contreexemple? Il n'y a, semble-t-il, que deux possibilités. On peut reformuler la définition en changeant la condition (v) de façon à faire une référence précise à un nombre limité de propriétés que possède l'incorporé, mais que ne peut posséder l'incorporant ; ou bien encore, ajouter à la définition une huitième condition qui permettrait d'exclure des cas comme ceux qui impliquent la montagne et la vallée.

6. La pente descendante de la montagne $m$ inclut la totalité d'au moins la surface extérieure de la montagne, qu'elle partage avec une partie propre de la pente ascendante de la vallée $v$. Mais la montagne $m$ n'est pas une partie propre de la vallée $v$, de sorte que le contre-exemple ne peut être infirmé par la clause (vii) de Margolis. Ceci découle du principe méréologique selon lequel pour n'importe quels particuliers concrets $x, y$ et $z$, si $x$ est une partie propre de $y$ et si $z$ est une partie propre de $x$, alors $z$ aussi est une partie propre de $y$. Supposons que $g$ soit une minuscule pépite d'or enfouie profondément au cœur de la montagne $m$ comme partie propre de celle-ci. La pépite $g$ n'est évidemment pas une partie propre de la vallée $v$. De par le principe exposé ci-dessus, la montagne $m$ ne peut être une partie propre de la vallée $v$, même si la vallée $v$ entoure entièrement la montagne $m$. 
La première méthode requiert la spécification des propriétés ou types de propriétés que possède l'incorporé, mais que l'incorporant ne peut posséder. La condition (v) de Margolis est censée être le fondement principal de la distinction, ou non-identité, entre incorporé et incorporant. Mais cette condition (v) exige seulement que l'incorporé possède quelque propriété d'une certaine sorte que l'incorporant ne peut posséder, et cette exigence n'est que trop facilement satisfaite dans le contre-exemple de la montagne et de la vallée. Si les haeccéités ou essences individuelles ne sont pas exclues de l'éventail des propriétés distinctives admises par la condition, alors il sera toujours possible de satisfaire banalement à la condition ( $v$ ) toutes les fois que la condition (i) sera satisfaite.

Pour combler cette brèche, la condition ( $v$ ) pourrait faire une référence précise à des propriétés intentionnelles ${ }^{7}$. C'est l'intentionnalité des expériences psychologiques et de la compétence linguistique des personnes que Margolis considère comme le fondement irréductible de la distinction entre les personnes et leurs incorporations matérielles. Et c'est l'intentionnalité de l'expression artistique, même pour ce qui concerne la sélection et l'exposition d'objets trouvés (found object) ou d'art tout fait (readymade art), qui est censée distinguer les œuvres d'art et les autres produits culturels de leurs incorporations matérielles. Si l'intentionnalité est irréductible à des propriétés physiques ou matérielles, alors elle est toute désignée comme type de propriété que possède un incorporé, mais que ne peut posséder son incorporant. Par conséquent, pour réviser la condition ( $\mathrm{v}$ ), considérons la formule suivante:

(v') Le particulier incorporé possède au moins quelques propriétés intentionnelles que le particulier incorporant ne peut posséder.

7. Margolis inclut les propriétés d'avoir des expériences sensitives ou phénoménales particulières dans la catégorie de l'intentionnel parce qu'il croit qu'un langage propositionnel intentionnel (tout autant qu'un langage intentionnel) doit être employé pour attribuer des expériences psychologiques à des êtres sensitifs autres que des personnes. Voir Persons and Minds, p. 187. Aussi MARgols, Knowledge and Existence: An Introduction to Philosophical Problems (New York : Oxford University Press, 1973), p. 243-244. 
Mais cette condition révisée peut s'avérer trop restrictive. Rien ne nous garantit que l'intentionnalité est la seule propriété irréductible susceptible de distinguer les particuliers incorporés émergents de leurs incorporations matérielles. Peut-être est-ce la raison pour laquelle, dans la formulation de (v), Margolis ne se compromet en faveur d'aucun ensemble précis de propriétés ou de sortes de propriétés.

Un autre argument contre ( $\left.v^{\prime}\right)$ a trait à l'émergence culturelle et à l'incorporation matérielle d'objets trouvés (found object) et d'art tout fait (readymade art) ${ }^{8}$. Ce type d'art acquiert son intentionnalité du dehors, lorsque des objets sont élevés au statut d'art par le fait qu'ils sont découverts, sélectionnés et exposés dans un contexte de conventions culturelles appropriées. Le rôle de l'artiste ici ne consiste pas tant à construire matériellement qu'à attirer l'attention sur des objets et à les étiqueter comme objets d'art, comme le fait Marcel Duchamp quand il prend le porte-bouteilles d'un fabriquant et crée son Bottlerack 9 .

Supposons qu'un artiste trouve par hasard la vallée $v$ contournant la montagne $m$ et qu'il décide d'entreprendre un projet à grande échelle d'objet trouvé ou d'art tout fait. L'artiste, symboliquement et à titre tout à fait gratuit, plante un drapeau coloré dans la vallée $v$; puis lui-même, les critiques et tout le milieu artistique se mettent à l'appeler la Vallée $V$. Ce faisant, il crée l'objet trouvé (found object) ou l'œuvre d'art toute faite (readymade artwork) Vallée $V$ à partir de la vallée $v$, tout comme Duchamp crée l'œuvre d'art toute faite Bottlerack à partir du porte-bouteilles du fabriquant. Ici, d'après la définition de l'incorporation avec la clause révisée ( $\left.\mathrm{v}^{\prime}\right)$, la Vallée $V$ émerge culturellement de la vallée $v$ et lui est matériellement incorporée, dans le sens où Margolis soutient que le Bottlerack de Duchamp émerge culturellement du porte-bouteilles du fabriquant et lui est matériellement incorporé.

Malheureusement, la Vallée $V$ et la montagne $m$ sont reliées de telle façon que, tout comme dans le premier contre-exemple, la

8. Art and Philosophy, p. 20-21.

9. Voir Arthur C. Danto, The Transfiguration of the Commonplace: A Philosophy of Art (Cambridge: Harvard University Press, 1981), pp. 93-94. Danto mentionne une interprétation similaire de Ted Cohen à propos de l'urinoir de porcelaine tout fait de Duchamp, Fountain. 
Vallée $V$ est elle aussi culturellement émergente de $m$ et lui est incorporée matériellement, d'après la définition de l'incorporation que propose Margolis avec la condition révisée ( $\left.v^{\prime}\right)$. La description et la justification de la satisfaction aux conditions de Margolis demeurent inchangées sauf pour la condition ( $\left.\mathrm{v}^{\prime}\right)$, quand on substitue «Vallée $V$ » à «vallée $v$ ». Pour satisfaire à ( $\left.\mathrm{v}^{\prime}\right)$ on peut prétendre qu'il existe au moins quelques propriétés intentionnelles que la Vallée $V$ possède mais que la montagne $m$ ne peut posséder. La Vallée $V$ possède la propriété intentionnelle d'être créée par un artiste en tant qu'objet trouvé ou œuvre d'art toute faite, tandis que la montagne. $m$ ne peut, en aucune manière, avoir cette propriété. (La Montagne $M$, semblablement créée par un artiste à partir de la montagne $m$ pourrait, on le conçoit, posséder cette propriété. Mais de par le concept d'émergence de Margolis, la Montagne $M$ n'est pas identique à la montagne $m$ pour la même raison que le Bottlerack de Duchamp n'est pas identique au porte-bouteilles du fabriquant) ${ }^{10}$.

La définition modifiée de l'incorporation entraîne que la Vallée $V$ est incorporée à la montagne $m$. Mais une partie seulement, tout au plus, de la Vallée $V$ est matériellement incorporée à une partie propre de la montagne $m$. Ceci démontre que la définition de Margolis, avec la condition révisée ( $\left.v^{\prime}\right)$, demeure sujette à un contre-exemple dûment reformulé. Il est tout aussi inexact et contraire à l'intuition de conclure que la Vallée $V$ est matériellement incorporée à la montagne $m$ qu'il l'est de soutenir que la vallée $v$ est matériellement incorporée à la montagne $m$.

L'autre méthode pour éviter ces contre-exemples consiste à ajouter une huitième condition qui éliminerait les cas déjà cités. Mais quelle nouvelle condition pourrait-on introduire? En ce qui concerne les contre-exemples de la montagne et de la vallée, la difficulté est que la vallée a des propriétés physiques ou matérielles que la montagne n'a pas. Ceci suggère la condition additionnelle suivante :

10. "The Ontological Peculiarity of Works of Art», p. 47-48; Art and Philosophy, p. 21 : "Quiconque dirait que le porte-bouteilles est identique au Bottlerack (le modèle ou le type) de Duchamp, serait en contradiction avec lui-même...» (Frank Jackson critique Margolis pour avoir rejeté l'identité en faveur de l'incorporation dans son ontologie des personnes et des œuvres d'art. Voir Jackson, compte rendu intitulé «Joseph Margolis, Persons and Minds», dans Noûs, Vol. XVII, 1983, p. 97.) 
(viii) L'incorporé ne possède aucune propriété physique ou matérielle qui n'appartienne aussi à l'incorporant.

La condition (viii) nous permet d'écarter les contre-exemples de la montagne et de la vallée, mais c'est là payer un prix trop élevé. Il existe des œuvres d'art et des personnes incorporées à des particuliers incorporants, et possédant des propriétés physiques ou matérielles qui ne sont pas aussi des propriétés de leurs incorporants.

Supposons qu'un artiste choisisse une pierre naturellement lisse comme support d'une miniature au fusain. D'après la définition de Margolis, le dessin est une entité culturellement émergente incorporée aux lignes et aux taches de fusain disposées sur la surface de la pierre. Le fusain ne peut flotter librement dans l'espace si on veut qu'il constitue un dessin, mais il faut qu'il adhère à une surface. On peut imaginer que les infimes rugosités, arabesques ou irrégularités de grain, sur la surface par ailleurs lisse de la pierre, déterminent jusqu'à un certain point la façon dont sortira le dessin, et sont responsables pour une part de sa beauté unique. Le dessin est alors matériellement incorporé au fusain et à la pierre, car sans celle-ci, il n'existerait pas. Il semble également exact de dire que cette ouvre d'art a (effectivement ou potentiellement) la propriété physique ou matérielle d'être totalement détruite lorsque le fusain est gratté de la surface de la pierre. Mais si l'incorporation matérielle du dessin consiste dans la pierre et le fusain, alors l'incorporant de l'œuvre d'art ne possède pas la propriété matérielle ou physique d'être totalement détruit lorsqu'on efface le fusain. La pierre, qui fait partie de l'incorporation matérielle du dessin, n'est pas détruite mais continue à exister sans changement important ${ }^{11}$.

Si l'esprit est mortel, alors certaines personnes, tout au moins, ont avec leurs incorporations matérielles un rapport précisément analogue à celui qui existe entre le dessin au fusain et

11. On pourrait affirmer que l'incorporation matérielle du dessin est complètement détruite quand le fusain est enlevé de la pierre. Mais ceci constituerait une condition peu plausible et excessivement rigoureuse de la destruction totale de l'incorporation du dessin, et qui susciterait des contre-critiques du type «bateau-de-Thésée». Il est généralement inexact de dire qu'un exposé entier est totalement détruit lorsque seulement certaines de ses parties propres le sont. 
son incorporation matérielle dans la poussière de fusain et dans la pierre. Les personnes, en tant qu'entités culturellement émergentes et matériellement incorporées, possèdent la propriété physique ou matérielle d'être totalement détruites dans toutes sortes de circonstances mortelles. Cependant, les incorporations matérielles des personnes n'ont pas nécessairement cette propriété, puisque souvent les corps humains continuent d'exister comme cadavres longtemps après la mort, ou la destruction totale, des personnes qui leur étaient incorporées.

Ceci montre qu'il existe des propriétés physiques ou matérielles appartenant à des entités matériellement incorporées, mais n'appartenant pas en même temps aux incorporants de ces dernières. La condition (viii) est donc inacceptable. Il se peut que d'autres conditions plus satisfaisantes soient utilisables, mais aucune ne semble sur le point d'apparaître. L'ontologie de Margolis concernant les personnes et les œuvres d'art pourrait en principe nous offrir un palliatif aux limites métaphysiques du dualisme ontique, ou platonisme, et du matérialisme réducteur. Mais son interprétation des personnes et des œuvres d'art comme entités culturellement émergentes et matériellement incorporées ne peut être acceptée à moins que, ou jusqu'à ce que, le concept d'incorporation matérielle soit adéquatement défini.

Department of Pbilosopby

University of Nebraska, Lincoln 